\title{
La clínica rural en el contexto indígena: alcances y desafíos
}

\author{
DANIEL FLORES ALBORNOZ
}

Este artículo analiza el desencuentro que suele existir entre las prácticas de las clínicas que operan bajo el concepto de interculturalidad en salud y la satisfacción de sus beneficiarios, en un contexto rural e indígena de México. Se analizan los alcances de la ciencia médica en la forma de vida local, tomando en cuenta que la gramática rigurosamente estructurada de la medicina suele impedir la comprensión de otros lenguajes. Se demuestra que la noción de interculturalidad en el sistema de salud pública, así como la relación salud/enfermedad, debe repensarse, con miras a favorecer una participación más abierta de la investigación interdisciplinar, ya que estos espacios públicos de atención a la salud se encuentran en contextos en los que se ponen en juego distintos lenguajes y formas de vida.

PALABRAS CLAVE: clínica rural, interculturalidad en salud, política pública, participación social, lenguaje y prácticas sociales.

\section{The Rural Clinic in Indigenous Contexts: Scopes and Challenges}

This article analyses the mismatch that usually exists between practices from clinics that work with the concept of intercultural health and the satisfaction of the beneficiary population in Mexican rural and indigenous contexts. The approaches that the medical science eventually have over the local forms of life are analysed, considering that medical science is a language with a rigorous grammatical structure, which limits the understanding of other languages. It is demonstrated that the notion of interculturality in the public health system must be rethought, as well as the health/disease relationship, in favour of a more open participation of interdisciplinary research, as these public spaces operate in contexts where very different languages and forms of life are put into play.

DANiel Flores AlbornOZ

Investigador independiente dfalbornoz@gmail.com
KEYWORDS: rural clinic, intercultural health, public policy, social participation, language and social practices. 
1 presente artículo surge de un estudio etnográfico en el que se analizan diversas modalidades de atención a la salud, tanto públicas como aquellas consideradas tradicionales indígenas, con el propósito de entender el impacto de la ciencia médica en la forma de vida local. El análisis, aquí, se restringe a una de estas modalidades: la clínica rural. Se toma como estudio de caso la clínica de Tanzozob, Aquismón, San Luis Potosí, que obtuvo su certificación en 2013, esto es, el reconocimiento por parte del sistema de salud como una clínica rural del Instituto Mexicano del Seguro Social (IMSS). ${ }^{1}$

Como parte de una política de inclusión social por medio de programas de salud pública en zonas rurales e indígenas del país, se crearon las clínicas rurales gerenciadas por el programa IMSS-Oportunidades — actualmente, IMSS-Prospera-, las cuales adoptaron el modelo de salud para pueblos indígenas y salud intercultural promovido por diversas organizaciones y agencias nacionales e internacionales (OPS, 1993; 2008; Almaguer, Vargas y García, 2014). ${ }^{2}$ Los esfuerzos han sido notorios en

1 Para el estudio de caso, a lo largo de un periodo de trabajo de campo de ocho meses, de agosto de 2014 a marzo de 2015, se llevó a cabo una serie de entrevistas con el médico residente, personal de enfermería, asistentes y promotoras de salud, y autoridades locales de los diferentes parajes desde los que la gente acude para recibir atención médica en la clínica, así como con pacientes y población que habita en las localidades beneficiarias. Asimismo, se hicieron ejercicios de observación de la atención, diagnósticos y tratamientos sugeridos, y de la dinámica social en torno a la clínica y la política pública de salud, desde la politización de los servicios hasta el movimiento de la economía en los mercados itinerantes, en relación con los pagos en efectivo de apoyos sociales, como, por ejemplo, las becas del programa Prospera. Este artículo es apenas un atisbo de todo lo que se pudo documentar. Para un análisis de las diversas modalidades de atención a la salud, véase Flores (2017).

$2 \quad$ La iniciativa de extender los servicios clínicos a la población rural e indígena tiene antecedentes en el Imss, desde 1974, cuando se instrumentó el Programa Nacional de Solidaridad Social, iniciativa que tomó fuerza cuando, en 1979, se creó el programa conjunto con la Coordinación General del Plan Nacional de Zonas Deprimidas y Grupos Marginados conocido como Imss-Coplamar, convertido en 2011 en el programa ImSS-Oportunidades. Véase IMSS (s.f.). La trasformación del programa Oportunidades en el programa Prospera se formalizó mediante decreto presidencial el 5 de septiembre de 2014. Véase Diario Oficial de la Federación (2014). Para una revisión de la trasformación de los programas de desarrollo social desde 1997 a 2014, véase Barajas (2016). Para un atisbo de la historia del desarrollo social como estrategia de gobierno en el marco del federalismo en México y el papel del programa Prospera en ese contexto, véase González y Zárate (2016). 
múltiples sentidos. No obstante, la noción de interculturalidad aplicada a la salud pública parte de una definición romántica de reconocimiento e intercambio de conocimientos, reciprocidad y simetría en las relaciones que no toma en cuenta que las mismas relaciones interculturales han propiciado relaciones dominantes a lo largo de la historia, y los estudios al respecto con frecuencia han excluido de los análisis las dimensiones diferenciales de "raza, clase e ideológicas” (Menéndez, 2006: 57), entre otros vectores que favorecen la desigualdad: "el objetivo es articular los procesos, programas, acciones y niveles de atención en las regiones indígenas, que permitan el acceso efectivo [a] los servicios de salud [...], en especial de los casos que requieren de una atención inmediata y oportuna" (Almaguer, Vargas y García, 2014: 88).

Como es evidente, la atención inmediata y oportuna sólo es posible en los términos de la ciencia médica. A pesar de los esfuerzos por contar con promotores locales que sirvan como traductores e intérpretes, y de que se estime que los médicos en turno reconozcan saberes y conocimientos locales, el encuentro con la ciencia médica en los diferentes niveles de atención implica el uso de lenguajes ajenos al paciente, sobre todo cuando los diagnósticos requieren tecnología sofisticada, que sólo es posible comprender mediante el uso del lenguaje científico apropiado. Por ello, no basta con pensar en un posible reconocimiento de la diversidad cultural, sino que es menester entender que la interculturalidad supone la mutua comprensión entre diferentes formas de ver el mundo.

Generar espacios de intercambio y abrir puentes que favorezcan la comprensión mutua parece no ser asequible por el simple hecho de que utilizar un lenguaje científico especializado requiere estudios académicos profesionales — de lo cual está excluida la mayor parte de la población-, y entender la forma de vida indígena implicaría conocer un conjunto de "juegos de lenguaje" ajenos a la ciencia médica y la vida cotidiana de los profesionales de la salud (Wittgenstein, 1999; Jacorzynski, 2008, 2010; Tomasini, 2014). Esto no quiere decir que la ciencia médica sea un lenguaje cerrado, de acceso imposible, o que la visión del mundo de algún grupo indígena sea incomprensible para alguien dispuesto a comprender. La complicación radica más bien en las reglas que dan sentido a uno u otro lenguaje en relación con sus formas de vida particulares. Visto así, la interculturalidad es básicamente una relación de aprendizaje, pero la ciencia médica no puede utilizarse con lenguajes diferentes, que no sean el propio lenguaje científico, pues no puede dejar de utilizar sus propias reglas gramaticales, tanto de semántica como de sintaxis. Esto quiere decir que las prácticas no científicas quedan fuera de la gramática del lenguaje médico y por ende son excluidas a priori. La interculturalidad, por lo tanto, se reduce a la búsqueda de respeto y tolerancia dentro del terreno de la ciencia, lo cual supone un lenguaje ajeno en gran medida a la forma de vida de la población a la que se intenta beneficiar. ${ }^{3}$

Podemos coincidir en que la noción de interculturalidad ha originado más confusiones que claridad en el análisis de las interacciones entre distintos grupos, como sugiere Néstor García Canclini (2011: 103). El hecho de que se haya reconocido la diversidad y con ello la desigualdad social ha sido un gran logro del siglo XX, pero la insatisfacción por el uso de esos términos, que dio paso al de interculturalidad, supuso un brinco analítico de riqueza a otro de desorden (García, 2011: 103). Así, la interculturalidad resaltó una serie de conflictos interétnicos, intergeneracionales e interinstitucionales. Además, hay que considerar que la interacción social trasciende lo inmediato, por medio de información mediática, en un contexto global en el que los receptores no son

Véase la definición de lenguaje de Ludwig Wittgenstein, en particular la de "juego de lenguaje" (1999: 7). 
entes pasivos sino que alteran sus propias concepciones y construcciones de sentido a partir de la información que reciben (Tomlinson, 2001), y como el propio García Canclini (2004) da a entender, los que estaban desconectados ahora están interconectados por medio de procesos desiguales.

La interculturalidad ha sido una buena categoría de análisis para entender los procesos de interacción social entre pueblos, naciones, instituciones, sistemas económicos y cualquier campo de acción que defina a un grupo social decidido a la interacción. Sin embargo, hasta ahora no se ha logrado definir espacios de salud pública en los que las relaciones sociales tengan la cualidad de reciprocidad y se respete de manera más equilibrada la forma de ver y entender de cada grupo, lo cual es un fenómeno de doble flujo. Los puntos plurales de encuentro no pueden eliminar las disparidades y relaciones asimétricas, aun cuando es posible ensayarlo en el nivel micro, en espacios cotidianos, como sugiere Gunther Dietz (2003). La propuesta pierde piso si se piensa en el contexto de la salud y el ejercicio de la medicina alópata en clínicas rurales. La interculturalidad implica que los profesionales de la salud inviertan tiempo para conocer diversas modalidades de atención en el contexto sociocultural local y ponerlas en práctica. Esto sitúa a los profesionales de la salud en un terreno desconocido, en el cual perderían el estatus de autoridad, pues la ciencia carece de valor absoluto. De igual manera, sería necesario invertir tiempo para que población local interesada en conocer el lenguaje y los instrumentos clínicos pudiese hacerlo, pero las clínicas rurales no pretenden ser centros de enseñanza y aprendizaje, menos aún de intercambio de saberes. La interculturalidad es entonces un discurso que adquiere sentido en la práctica, pero sin dejar de ser discurso. Desde este punto de vista, la interculturalidad es una paradoja.

Lo que comúnmente se considera lineamiento intercultural en salud consiste en una serie de valores universales. Por ejemplo, en un cartel informativo en la clínica de Tanzozob se señala lo siguiente:

Lineamientos de la interculturalidad: reciba al paciente con un saludo respetuoso. Explique claramente la enfermedad, tratamiento y cuidados. Evite burlarse, regañar o prohibir. En la atención del parto permita la deambulación alternada con reposo. Establezca con el o la paciente, familiares y acompañantes actitudes respetuosas de comprensión y amabilidad. Evite emisión de juicios, prejuicios. Fomente la participación social y la comunicación asertiva. Distinga características e identidades culturales. Revise al paciente previo su consentimiento.

Asimismo, se destacan las obligaciones que tiene el personal de enfermería, publicadas también en la clínica:

1) Respetar y cuidar la vida y los derechos humanos, manteniendo una conducta honesta y leal en el cuidado de las personas. 2) Proteger la integridad de las personas ante cualquier afectación, otorgando cuidados de enfermería libres de riesgos. 3) Mantener una relación estrictamente profesional con las personas que atiende, sin distinción de raza, clase social, creencia religiosa y preferencia política.

Esta serie de lineamientos no se diferencian realmente de los derechos básicos de cualquier paciente, con independencia del contexto social, pues son derechos universales que se basan en el respeto y la mutua comprensión. El problema radica en cómo lograr una verdadera comprensión entre dos o más partes que interactúan por medio de lenguajes y formas de vida distintos, puesto que la tendencia es que la comprensión de la ciencia médica, así como la política que hace operativos a los centros de trabajo, quede restringida al gremio.

Sergio Lerín (2004) observa que el uso de la interculturalidad en salud sirve como herramienta del 
poder para opacar las desigualdades sociales y que, en efecto, existe la necesidad de profesionalizar al personal clínico en relación con contenidos y contextos interculturales. En los centros de salud, clínicas, hospitales regionales y unidades médicas móviles, los expedientes de atención a la salud de la población indígena reportan el diagnóstico de las enfermedades atendidas; sin embargo, no existen reportes que se refieran a "la percepción indígena del proceso saludenfermedad-atención, ni tampoco se ocupan por dar cuenta de los eventos atendidos al margen de los sistemas institucionales de salud" (2004: 115). Lerín también señala la necesidad de una articulación del dato clínico con la dimensión sociocultural para entender cómo se interrelacionan los distintos sistemas de conocimientos en temas como el diagnóstico y la atención. Esto revierte el enfoque y sitúa a la medicina alopática como complementaria de los sistemas de salud indígenas, lo cual implicaría generar una nueva especialidad médica. ${ }^{4}$

Hay un punto de tensión entre los sistemas clínicos alópatas y los sistemas de atención a la salud en el marco cultural indígena. Considérese, por ejemplo, el hecho de que en el país existe una prevalencia importante de las llamadas "enfermedades de rezago" (Lerín, 2004: 114), como el dengue, la tuberculosis, el paludismo, entre otras, contra las que el conocimiento de la ciencia médica contribuye con probada efectividad. Además, cuando la atención clínica demuestra su efectividad, trastoca la forma de vida local al modificar algunas prácticas cotidianas, lo que cuestiona la forma de vida que se tiene, o que se busca, a la luz de los valores culturales de los pueblos originarios. Por lo tanto, vale la pena analizar la posición que ocupan los grupos indígenas en la atención definida por las instituciones de salud pública.

Lerín (2004: 117) ubica tres tipos de intervención: "la interculturalidad como movilización, la interculturalidad como gestión y la interculturalidad como reciprocidad". La movilización responde a una intervención externa, como ocurre en una clínica del IMSS. La gestión se resume en la participación de agentes locales que sirven de intermediadores, como promotores del lenguaje científico médico capaces de persuadir en la lengua indígena nativa. La reciprocidad señala la construcción de espacios en los que se encuentran médicos alópatas con médicos indígenas tradicionales, como ocurre en los hospitales mixtos, aunque esto suele ser ineficaz en tanto que se reproduce un tipo de "interculturalidad asimétrica” (Zolla, 2011). De manera que las iniciativas interculturales se han limitado al análisis y la revaloración de la cosmovisión a partir de conceptos como cuerpo y persona, las terapéuticas mágico-religiosas, la organización social comunitaria en torno a la salud/enfermedad, entre otros temas de orden cultural, como los mitos fundantes, el curanderismo, los destinos del alma después de la muerte y la relación que se establece con los ancestros muertos (López, 1972; 1984; Broda y Báez, 2001; Gallardo, 2004; Gallardo, 2005; Flores, 2012).

Por otra parte, existen estudios que abordan la salud como un problema estructural, en el que

4

Las innovaciones científicas demuestran que la ciencia, no es un lenguaje cerrado incapaz de comprender otras formas de conocimiento. Un ejemplo interesante lo constituyen las tomografías cerebrales a monjes budistas en estado de meditación y personas comunes para su comparación. Esto para demostrar científicamente el comportamiento del cerebro y los beneficios de la meditación budista para la salud física y mental. Existen varias referencias al respecto en revistas arbitradas de Estados Unidos y otros países, pero para una consulta general puede recurrirse a la página web de Mind and Life Institute, disponible en: <https://www.mindandlife.org>. El desafío, en todo caso, es para el contexto indígena mexicano: cómo hacer para que la ciencia médica pueda encontrarse de mejor manera con las prácticas de curación locales. La iniciativa de Lerín (2004) tiene algo relevante a este respecto, aunque no sea la interculturalidad lo que resuelve el problema, al menos en términos del lenguaje y sus gramáticas correspondientes. 


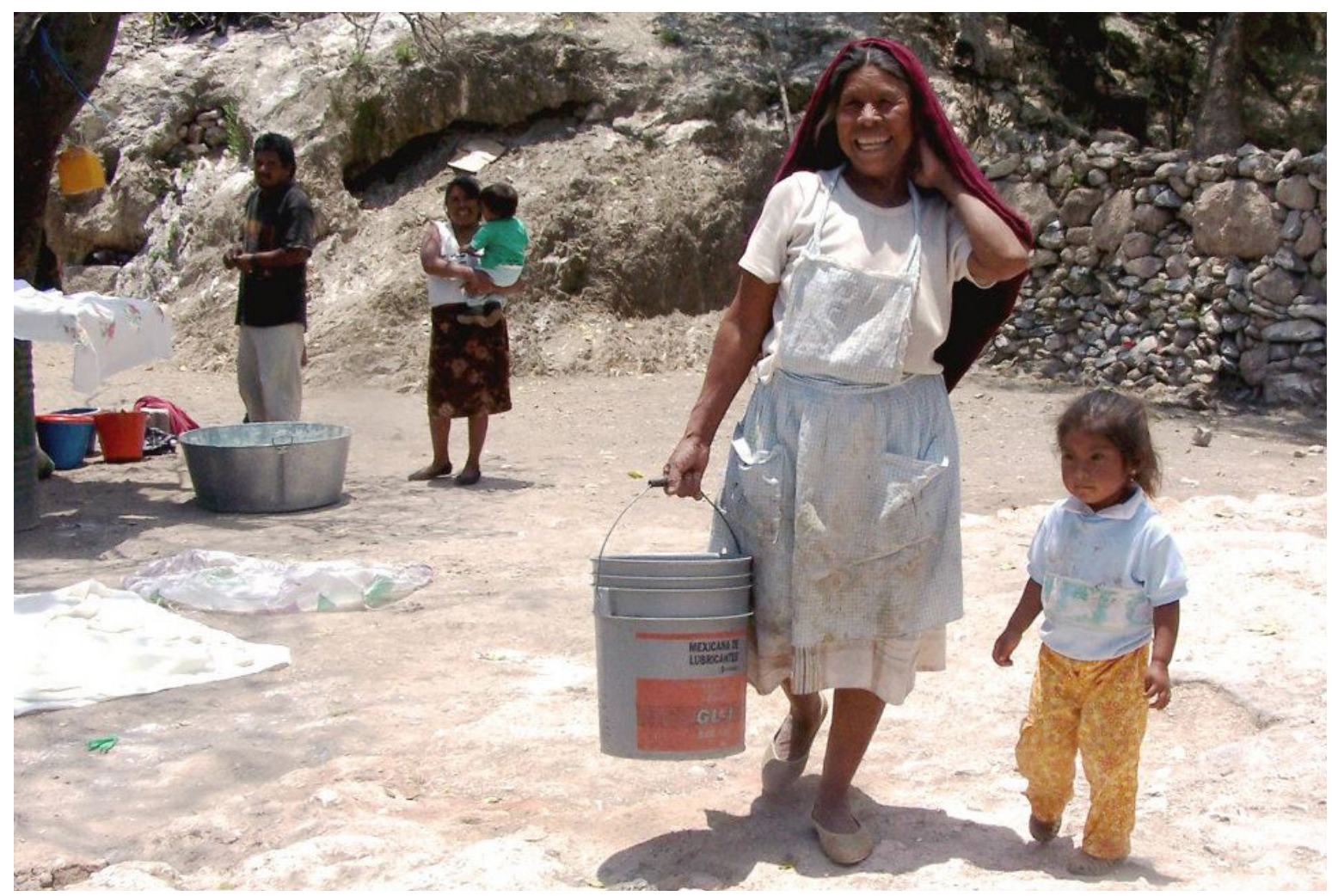

Verónica A. VelÁzQuez • Vida cotidiana de la comunidad hñahñu de Tolimán, Querétaro.

la dominación se manifiesta en el ámbito del conocimiento, el manejo de la información y la construcción de sociedades de riesgo, en las cuales la salud se presenta de manera frecuente como una catástrofe (Menéndez y Di Pardo, 2009). O bien, piénsese en la idea de la calidad de vida, que se asocia a la moral y el consumo de bienes materiales, más que a hábitos de vida saludables. O los análisis sobre enfermedades que se definen desde la geopolítica y la pretensión de homogeneización global de la cultura, cuyo efecto es la marginación de culturas distintas a la occidental, como en el caso del VIH/Sida asociado a comunidades africanas a finales del siglo XX y principios del XXI (Comaroff y Comaroff, 2013).

Desde esta perspectiva, la interculturalidad como política de atención a la salud se restringe al respeto que se ofrece al paciente por sentido común, mientras tanto, el lenguaje científico domina las prácticas de atención y se mantiene un distanciamiento sistemático con otro tipo de lenguajes. Lo que se demuestra aquí es que dicho distanciamiento es la constante, en tanto que el lenguaje científico no tiene traducción ni las curaciones espirituales pueden ser demostradas mediante el lenguaje científico médico. En un sentido estricto, no es posible interculturalidad alguna en esta situación, de modo que el concepto es útil para reconocer diferentes formas de vida pero no para analizar las prácticas de atención a la salud si se toman en cuenta las diferentes modalidades que en ocasiones se ponen en práctica de manera simultánea (Flores, 2017). El hecho de que existan diferentes modalidades de atención simultáneas no implica que haya una comprensión mutua entre ellas. Queda pendiente, además, demostrar que el uso de 
objetos producidos por la tecnología científica y farmacéutica en prácticas de cosmovisión indígena, como, por ejemplo, el uso de inyecciones u otras prácticas en terapias de curación espiritual indígenas, no supone una relación intercultural, como sugiere Eduardo Menéndez (2016: 112), a menos que se elimine de la observación etnográfica la dimensión del lenguaje, es decir, los significados y prácticas sociales que se generan a partir de la definición de conceptos, los cuales, a su vez, refieren a formas de vida como un conjunto coherente y complejo. Cuando un concepto pierde su sentido al aplicarse en un contexto distinto al original, no puede asegurarse que haya cierto tipo de continuidad cultural. ${ }^{5}$

\section{Representaciones sociales y el anhelo profesional de generar conciencia}

Durante el trabajo de campo tuve la oportunidad de grabar una serie de entrevistas que revelan un conjunto de representaciones sociales que han tomado forma a lo largo de la historia y que hoy en día se han naturalizado. Con representaciones sociales me refiero a la imagen subjetiva que se construye, tanto de manera individual como colectiva, sobre un grupo social. En este caso, la imagen subjetiva del médico residente sobre la población indígena desde su propia noción de salud y a partir del lenguaje científico que caracteriza la formación profesional. Destaca, por ejemplo, el hecho de que no se pueda concebir que algunas personas no acudan a los servicios de atención y queden al margen de los programas gubernamentales:

La otra [dificultad] es que muchas personas no se acercan a nuestras instituciones, a pesar de que nuestra institución no cobra un solo peso por ningún servicio [...], a nivel federal hay muchos programas $[\ldots]$ pero desafortunadamente hay personas que no los aceptan. Por ejemplo, una señora viene descalza y le digo: "oiga señora, ¿qué, usted no tiene Oportunidades?”. "No". "Pero, ¿no le interesaría entrar al programa? Mire que le va a llegar un recurso, con eso usted va a poder comer mejor, va a tener para comprar zapatos". "No me interesa". "Oiga, pero mire que le va a ayudar". "No me interesa” (entrevista con Felipe González, médico residente, Tanzozob, Aquismón, San Luis Potosí, 28 de julio de 2014).

La idea de que los servicios de atención pública son universales supone una regla lógica en el pensamiento institucional, lo que por principio contradice la idea de que alguien tome la decisión de no recibir los beneficios de los programas sociales. El ejemplo revela, por un lado, la capacidad de las instituciones públicas de convertir a la persona en objeto de beneficio social, y por el otro, la posibilidad real de que ciertas personas decidan no recibir apoyos gubernamentales y con ello invertir la lógica de la exclusión social. Esto significa que la persona se convierte en excluida por decisión propia, por lo tanto, la exclusión desde la institución es, al parecer,

Un ejemplo interesante es el concepto de coup-stick en la antigua cultura crow, al norte de Estados Unidos. El coupstick - la vara ritual del guerrero- caracterizaba la forma de vida crow en su conjunto. De este modo, el mismo concepto podía definir una serie de prácticas sociales, como la delimitación del territorio mediante fronteras simbólicas en advertencia al enemigo, o un conjunto de categorías de valor social, como el honor, el estatus jerárquico del guerrero dentro del grupo y hasta la preparación de alimentos. En el momento en que los crow quedaron confinados a una reserva territorial, el concepto coup-stick perdió su significado, y aun cuando dentro de la reserva se pudiesen recrear rituales asociados a la vara ritual del guerrero, el uso original dejó de existir, por lo cual no se puede decir que en la actualidad se utilice el concepto de coup-stick en un contexto intercultural. Cuando un concepto carece de sentido al utilizarse en un contexto distinto al original, entonces deja de ser el concepto que era, y por lo tanto, no es garante de la continuidad cultural. Al respecto, véase Lear (2006). 
inválida. Desde la lógica institucional, no hay justificación para negar una ayuda gubernamental, por lo mismo, ese tipo de decisión puede considerarse irracional. Lo irracional, en este caso, se representa en el hecho de andar descalzo. ${ }^{6}$

Podría pensarse en diferentes formas de exclusión por decisión personal en relación con la política en México, por ejemplo, cuando alguna persona no se interese en obtener su credencial para votar ni en participar en la elección de sus gobernantes. Sin embargo, eso no es equiparable a la posibilidad de obtener un apoyo económico y no aceptarlo. La noción de desarrollo social asociada a la idea de pobreza tiene un peso moral de importancia entre los servidores públicos, muy distinto al de la credencial para votar, excepto entre quienes están interesados en obtener votos. La autoexclusión, que podría entenderse como una forma de resistencia o de práctica de la autonomía, cuando está en relación con la salud pública, no se trata tan sólo de una posición política, sino, como comenta el médico, de una falta de conciencia entre la población, para que la clínica, como extensión del desarrollo social y la salud comunitaria, impacte de mejor manera:

Me tocó un caso [...] de un paciente con deshidratación [es jornalero migrante]. Entonces dura allá un mes, más o menos [...]. Se van, mal comidos, [con] exceso de trabajo, [sufriendo] el calorón, todos cansados. Le expliqué [la situación] a la auxiliar de la comunidad donde vive el muchacho. A la semana, ya me lo mandaron temporalmente [para administrarle suero intravenoso]. Entonces, pues, todavía falta [...] concientizar a la población sobre los problemas de salud y que ellos nos comprendan, que no estamos en contra de la medicina tradicional, pero que la medicina tradicional, al igual que nuestra medicina, tiene sus límites, y [...] cuando ese límite ya pone en peligro la integridad de la persona, pues sí, se tiene que buscar otra alternativa. [Por ejemplo], un paciente con un absceso [que] principalmente le genera un dolor en el hombro derecho, y nadie en las comunidades le va a decir qué tiene realmente. El señor dice: “¡me duele el hombro!”. Y tiene dolor de estómago y todo. No tiene lo típico porque tiene sangrado al evacuar. Entonces le digo que es necesario hacer un ultrasonido, le doy un pase para que vaya al hospital regional. Pero antes de eso él estaba pensando que le estaban haciendo brujería (entrevista con Felipe González, médico residente, Tanzozob, Aquismón, San Luis Potosí, 7 de agosto de 2014).

La deshidratación no atendida en el ámbito doméstico es un ejemplo interesante de lo simple que puede ser comprender situaciones que afectan la salud personal y la necesidad de difundir un mayor conocimiento clínico al respecto. Si la persona no comprende el concepto de deshidratación, pues éste no forma parte de su forma de vida, y por el contrario, en el contexto del curanderismo se le dice que tiene un "aire de muerto" (Flores, 2017); la deshidratación puede avanzar al grado de necesitar suero intravenoso. La idea de conciencia, desde este punto de vista, se restringe al uso de un lenguaje de carácter científico que pueda ser incorporado a la realidad cotidiana de personas que se desenvuelven en ámbitos culturales ajenos a los de la clínica rural, aun cuando la clínica es un espacio con el cual se convive con frecuencia. Es un problema de conceptos y lenguajes, más que de falta de conciencia. Incluso si el concepto

$6 \quad$ La definición de pobreza que subyace en las instituciones públicas que ofrecen atención a la población considerada vulnerable y que habita en zonas de atención prioritarias es la que propuso el Consejo Nacional de Evaluación de la Política de Desarrollo Social (Coneval, 2019: 33): "una persona se encuentra en situación de pobreza multidimensional cuando no tiene garantizado el ejercicio de al menos uno de sus derechos para el desarrollo social, y si sus ingresos son insuficientes para adquirir los bienes y servicios que requiere para satisfacer sus necesidades". 
de clínica y sus prácticas en relación con el lenguaje médico se encuentran disponibles en los diferentes espacios de interacción social y cultural, sólo a partir de la experiencia se puede comprender dicho concepto. Es probable, por ejemplo, que el concepto de deshidratación empiece a adquirir sentido en la persona a quien le fue administrado el suero después de haber sido internado, sin embargo, eso no necesariamente cambia la probable causa vinculada a un aire enfermizo. La experiencia dentro de la clínica, entonces, es el espacio en el que se empieza a generar cierto tipo de conciencia, en tanto que obliga a utilizar conceptos de carácter científico aplicados a una situación personal. ${ }^{7}$

En este sentido, la educación en salud mediante pláticas no es suficiente, y muchas veces tampoco lo es obtener grados escolares. En la región de estudio, por ejemplo, es frecuente ver personas que han egresado de algún bachillerato acudir con curanderos como primera opción, y después a la clínica, para obtener medicamentos; si los medicamentos alópatas no eliminan el padecimiento, entonces confirman un diagnóstico de brujería. Paradójicamente, la política intercultural en salud puede potenciar esta confusión, antes que corregirla, cuando los diagnósticos que se establecen no son del todo certeros o cuando tienden a ocultarse males mayores si los propios pacientes no regresan más para dar seguimiento o descartar alguna otra causa probable. Por otra parte, la prevalencia de enfermedades que requieren atención clínica es alta. Entre los casos que fueron diagnosticados durante el periodo de trabajo de campo, se pueden mencionar algunos: "un tumor a nivel uterino, mioma, precisamente [...]. Otro fue un paciente con un problema neurológico, una tumoración a nivel meninge [...]; un caso es de tuberculosis" (entrevista con Felipe González, médico residente, Tanzozob, Aquismón, San Luis Potosí, 7 de agosto de 2014). Cabe señalar, además, que la mayor parte de la población de más de 30 años de edad padece diabetes e hipertensión.
El médico señala, con mucha razón, que en cierto modo la conciencia sobre la salud y la enfermedad tiene que ver con reconocer los límites de las diferentes formas de atención, y por lo tanto, acudir a la clínica a tiempo para atenderse. Esto es un círculo vicioso: reconocer los límites conceptualmente es imposible a menos que se comprendan los conceptos de diferentes lenguajes que no son comunes entre sí, pero para empezar a comprender los conceptos de la práctica clínica hay que presentarse en calidad de paciente, en busca de la atención que el curanderismo o la medicina indígena tradicional no han podido solventar. Al enfrentarse a conceptos que carecen de sentido, se produce confusión y se recurre de nuevo a la brujería como explicación causal de la enfermedad, o bien, se reconoce que existen factores causales que tienen una explicación científica, y aun cuando no se lleguen a comprender a fondo los conceptos, se cuestiona el curanderismo y se reconoce que existen límites en las formas de atención consideradas tradicionales. Es comprensible, desde este punto de vista, que la noción de interculturalidad se reduzca al respeto

Conviene distinguir diferentes tipos de conceptos. Algunos, como jeringa, venda, pastilla, etc., se comprenden con facilidad puesto que su significado se adquiere a partir del uso que se da a dichos objetos. Pero conceptos más abstractos, como diabetes, hernia o aquellos que surgen de diagnósticos clínicos sofisticados, son difíciles de comprender puesto que no tienen un uso concreto en la forma de vida cotidiana. Aquí, la frontera entre los profesionales de la salud y los pacientes legos toma forma, ya que, aun cuando el paciente pueda observar una tomografía o leer los análisis de laboratorio, los conceptos carecen de un sentido útil. Cuando el curanderismo no puede atender problemas de salud de esta naturaleza, y cuando la ciencia médica es poco comprensible, cabe la posibilidad de entrar en confusión pues la forma de vida que se tiene o que se busca carece de sentido. Este fenómeno es digno de estudios más acabados, porque se puede interpretar como un efecto negativo de la ciencia médica, que modifica la forma de vida local sin que la población se apropie de una nueva forma de vida bien definida y socialmente reconocida. 
que por sentido común se ofrece a cualquier persona y que de ahí en fuera sea un concepto vacío o en todo caso una iniciativa de carácter político, sin que tenga un impacto real en lo que el médico considera "generar conciencia". De este modo, el impacto negativo de la ciencia médica origina insatisfacción profesional. Como dice el médico, no hay manera de obligar a la población local a comprender el lenguaje de la medicina.

Busqué entonces un caso de contraste durante el trabajo de campo. En una localidad de alrededor de 200 habitantes, circunvecina a Tanzozob y que recibe atención de la clínica, se había presentado un caso de deshidratación severa en un bebé de apenas seis meses de edad. Acudí al domicilio acompañado de la auxiliar de salud local. Resultó ser el domicilio de una señora de alrededor de 40 años de edad, su hija de tan sólo 17, a la que identificaremos como A., y el bebé de A. Nadie más vive con ellas. El domicilio, compuesto de dos pequeños cuartos - uno que funge como cocina y otro como dormitorio-, con piso de tierra, paredes de otate y techo de palma, dejaba entrever el aislamiento y la pobreza en los que vivían, en términos de desarrollo social. A. quedó embarazada de alguien aparentemente desconocido. Ella es sordomuda, y por su condición, alguien al parecer abusó de ella cuando estaba sola en casa, con lo que quedó embarazada. Ella y su familia no reciben ningún tipo de apoyo económico de los programas sociales como Prospera, pues no se han afiliado. ${ }^{8}$

Pude entrevistar a la madre de A., mientras ella, sentada en un rincón de la cocina, intentaba dar pecho a su bebé. La madre, en un español con dificultades, explicaba la situación. A simple vista, A. presentaba síntomas de desnutrición. No había mucho que pensar, le hacía falta alimento para poder a su vez alimentar al bebé. ¿Serviría de algo, pensando en un tratamiento clínico inmediato, administrar suero oral al bebé? Probablemente, pero eso no resolvería el problema de desnutrición del bebé ni de su madre.
Esta situación se inserta entre los temas sobre la marginación social generada, ya no sólo por una cuestión de carácter macroestructural, sino por su efecto en el nivel micro, entre los vecinos de una comunidad muy pequeña. El lenguaje de la política de desarrollo social, al parecer, divide a las familias porque, si los programas gubernamentales están para ayudarles, vivir en la pobreza extrema se interpreta como una decisión propia.

Pero entonces, ¿por qué esta familia no recibe apoyo de algún programa gubernamental? Porque ellas no participan en las reuniones; A. no asiste a ningún plantel escolar, y aparentemente, no forman parte de la organización comunitaria que podría canalizarlas para que se les otrogue algún apoyo. ¿Qué sentido puede tener el suero oral para una familia que se encuentra en los márgenes del sistema y su alimentación básica es deficiente por falta de recursos económicos? Desde esta perspectiva, el suero oral, como cualquier otro medicamento, no es otra cosa que un "calmante", como lo denomina la población local, que no cura el problema de fondo, y menos aún cuando es un problema de orden económico que deviene en un problema de salud.

Desde la perspectiva del médico residente, el esfuerzo de "incluirse" para recibir algún beneficio gubernamental implica también "abrirse", esto es, asumir las responsabilidades que conlleva participar en cada programa y hacer a un lado la "cerrazón”, que no permite acceder a los beneficios que pueden, a su vez, reflejarse en la salud. Esta "cerrazón” se explica, según el profesional, por la apatía de la gente:

En ese sentido, la apatía de las personas de las comunidades es lo que ha hecho que impacte de manera

8 La situación revela, en términos legales, un acto de violencia sexual con el agravante de que A. padece una discapacidad auditiva y era menor de edad al momento del acto. Esto desvela el grado de normalización de la violencia en este tipo de contexto social, tema que queda por el momento como una limitante del presente análisis.

La clínica rural en el contexto indígena: alcances y desafíos 
negativa en su salud y es algo que tiene que ver con todo el tema de desarrollo social. Por ejemplo, como el caso de una señora que se fue de su casa, dejó a los niños, tenía una tumoración a nivel de la pierna derecha. Para empezar, no tiene acta de nacimiento, por lo tanto, no puede acceder al Seguro Popular. Uno trata de buscar el procedimiento y las instancias que le ayuden a tramitar su acta y que pueda acceder al Seguro Popular, pero no aceptó. Entonces, lo que se hace es un llamado y se avisa al DIF [Sistema Nacional para el Desarrollo Integral de la Familia], y ya ellos tienen una facultad jurídica que nosotros no tenemos (entrevista con Felipe González, médico residente, Tanzozob, Aquismón, San Luis Potosí, 28 de julio de 2014).

Para que la clínica pueda ofrecer un mejor servicio, no basta con que obtenga una certificación y posea mejor equipo y mejores instalaciones en general, pues la población "cerrada" es una limitante, y en ocasiones es necesario actuar con base en la fuerza jurídica. Por esta razón, no tiene sentido hablar de una política y menos aún de una atención intercultural a la salud. Por mucho respeto que se tenga hacia las personas, si éstas deciden mantenerse al margen de las instituciones, no hay cómo ayudarles. No se trata, como leemos en el testimonio del médico, de un problema de carácter institucional, sino de un problema de falta de conciencia por parte de quienes podrían beneficiarse mejor de los programas sociales. Sin embargo, como se observa en el siguiente caso, parece que la distancia entre la ciencia médica y la población beneficiaria no se limita a estar o no al margen del sistema y los programas sociales.

\section{El encuentro inminente con la clínica rural}

El caso de Nazaria era atípico. Requería atención médica de urgencia de tercer nivel pero el traslado a la capital del estado se había complicado. Sus padres se mostraban renuentes al traslado, pues consideraban que las causas de su problema de salud eran de orden espiritual y su prioridad era encontrar remedio mediante la atención de curanderos con cierto grado de especialización. El padre era curandero y él mismo hacía todo lo que estaba a su alcance para reestablecer la salud de su hija. Los padres, los profesores, las autoridades locales y el propio médico residente manifestaron su preocupación por este caso, pues la vida de la adolescente, de 15 años de edad, estaba en peligro. El malestar de Nazaria comenzó con un vómito que le afectó notablemente, ya que durante dos semanas no logró aprovechar bien sus alimentos, hasta que finalmente la llevaron a la clínica rural en calidad de urgencia. Al parecer, tardaron tanto en acudir a consulta porque la madre no quería que Nazaria dejase de asistir a la Telesecundaria, por temor a perder el apoyo de Prospera. Según el médico, la situación de la paciente era la siguiente:

A la hora de revisarla pues ya vemos que tiene un problema cardiaco. Aquí lo especial del caso es que es un problema congénito, que no se había manifestado en ninguna etapa. Ahorita tiene 15 años y anteriormente no tenía sensación de falta de aire, que se agitara cuando corriera, nada. Aparentemente, todo era normal. [Se le canalizó al hospital regional]. Allá le detectaron una cardiomegalia severa. Entonces, le detectan una insuficiencia cardiaca, pero le determinan que tiene una persistencia del conducto arterioso desde nacimiento. Aquí lo atípico es que habitualmente esta patología se manifiesta en los primeros meses de vida. En cambio, la paciente no desarrolló la sintomatología y su cuerpo tuvo la capacidad para mantener un equilibrio [...]. El corazón, como aparece en la placa, abarca casi la totalidad del pecho. Uno ve y toca a la paciente y parece que el corazón se le va a salir [...]. El problema que encontramos fuerte fue que la detectamos, la canalizamos a segundo nivel, allá hicieron 


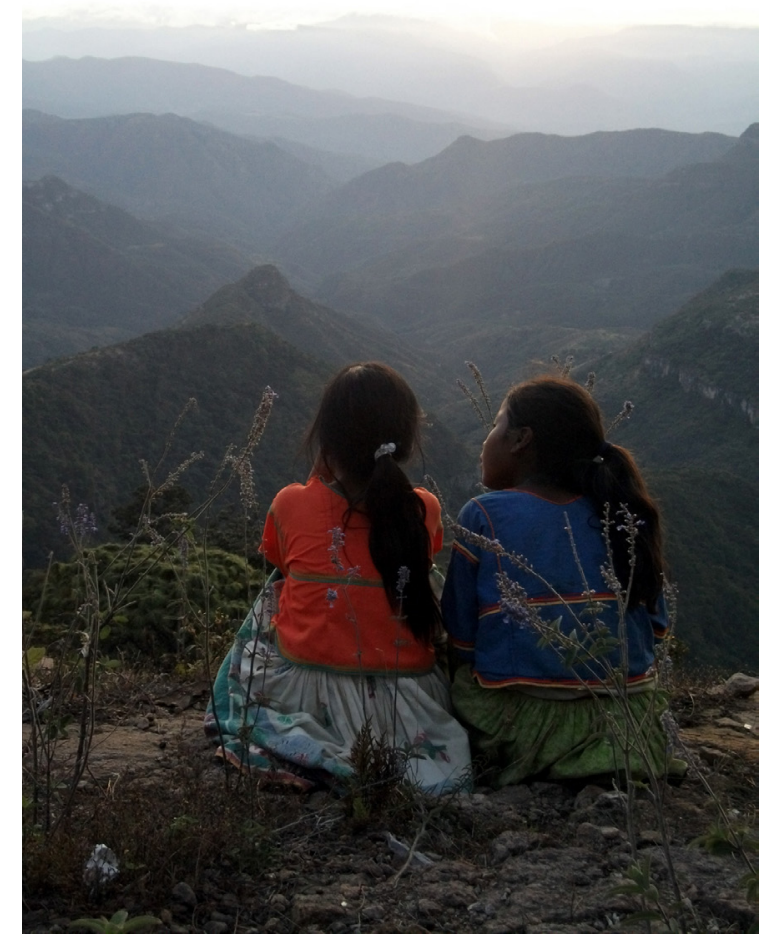

Gabriel CabezAs Atardecer en la sierra. Mezquitic, Jalisco, México.

lo propio y la canalizaron a tercer nivel, pero ¿cuál fue el detalle? La mamá no estaba muy convencida del manejo [traslado a la ciudad]. Ella [Nazaria] tenía la cita para atenderse el día 29, pero como el día 29 [la madre] tenía la reunión para recibir el apoyo de Prospera, pues no acudieron a la cita (entrevista con Felipe González, médico residente, Tanzozob, Aquismón, San Luis Potosí, 12 de agosto de 2014).

La necesidad económica se combinó con el temor a que se llevasen a Nazaria a una ciudad situada a alrededor de ocho horas de distancia en vehículo, con el riesgo latente de que muriera en el hospital, lejos del entorno familiar. Sin embargo, la preocupación familiar no residía solamente en los padres, pues la hermana, quien funge como promotora de salud local, intervino para convencerlos de trasladarla a la capital del estado. Surgieron entonces diferentes versiones. La hermana argumentó que el retraso del traslado se debía a cuestiones de carácter institucional, pues el vehículo dedicado a los traslados de urgencia no estaba disponible y no había llegado a la cita programada. El hecho se confirmó cuando la juez del paraje buscó ayuda entre sus contactos de la capital del estado para tratar de solventar el traslado. En el ámbito local no había mucho que hacer, según el diagnóstico clínico, excepto administrar medicamentos para el dolor y las náuseas y evitar una deshidratación mayor.

Mientras tanto, los padres hacían limpias espirituales, y al realizar un diagnóstico, entre otras cosas, al barrer a Nazaria con un huevo y escobas de diferentes yerbas, determinaron que los difuntos la estaban vigilando, y por lo tanto, corría riesgo de muerte. La posibilidad de muerte se diagnosticó porque, al romper el huevo en un vaso con agua, alrededor de la yema aparecieron muchas burbujas. Las burbujas representaban los ojos de los muertos. El tratamiento implicaba una serie de limpias espirituales, el uso de medicamentos a base de yerbas, rezos y una serie de ofrendas. Era necesario "barrerle el mal sombra”, como me explicó la madre. Para ello, todas las noches a las nueve en punto, durante nueve veces, se le hacía una barrida espiritual. La escoba para barrer "mal sombra" se hace con añil y yerba verde - esta última es un tipo de epazote de monte- y se limpia tanto el cuerpo de la enferma como el ambiente del hogar, quemando copal. Durante el día, se le aplicaban baños de agua caliente con árnica y micle, la primera para desinflamar y la segunda para desintoxicar el cuerpo, pero Nazaria se encontraba muy débil y se había convencido a sí misma de que era un problema para la familia, por su condición. Si no había solución para su problema, prefería morir.

Días después se consiguió un vehículo para trasladar a Nazaria, pero la madre, quien estaba renuente al viaje, quedó decepcionada pues el vehículo sólo llegó hasta la cabecera municipal y tuvieron

La clínica rural en el contexto indígena: alcances y desafíos 
que regresar por su cuenta, lo que implicó un gasto innecesario. Finalmente, tras varias semanas de espera y desesperación, se consiguió el traslado hasta el hospital central y Nazaria fue internada. Logró estabilizarse y pasó la emergencia. La atención clínica y hospitalaria había logrado su cometido y se salvó así una vida en riesgo. Pregunté entonces por la importancia de realizar investigación científica, dado lo atípico del caso, a lo cual el médico respondió lo siguiente:

Bueno, obviamente es algo emocionante porque es un caso que puede ser uno en un millón. A nivel científico o médico; es interesante, pero a nivel emocional, ahí está la otra parte del impacto al interior de la familia, pero más que nada, de la paciente [...]. Todavía recuerdo cuando llegó a verme la primera vez, con su mamá; la resistencia a querer revisarse en la clínica, y al final, pues bueno, se animó; y sí lo pensé, porque a veces manda uno al hospital y ya no atienden, preguntan que para qué mandan porque consideran el diagnóstico del médico general como ya válido. Pero ya cuando regresó [...], venía con todo el protocolo de estudio para atención a tercer nivel; es un caso que no deja de sorprenderme (entrevista con Felipe González, médico residente, Tanzozob, Aquismón, San Luis Potosí, 12 de agosto de 2014).

A pesar del interés del caso en términos científicos, llama la atención que el médico haya puesto como factor de importancia el tema ético antes que el científico. Esto es comprensible porque el logro de la intervención clínica rural consiste en canalizar a la paciente. Es decir, el logro médico se encuentra más en la gestión que en la investigación científica, lo cual demuestra que el quehacer científico es en realidad una práctica ajena al quehacer médico de una clínica rural, en tanto que la práctica médica se reduce a un trabajo técnico, aun cuando se requiere una formación profesional bastante sólida para ello. Aquí la ciencia médica sirve para establecer un proceso de formación profesional, y a partir de ahí se adquiere un lenguaje y una forma de vida, pero la ciencia, entendida como producción de conocimiento, desaparece, y la práctica queda restringida a la operación técnica.

De esta manera, la ciencia médica se reduce a un trabajo de orden técnico, en el marco de una política pública; mientras que el quehacer médico, en una clínica rural, deja de hacer ciencia — como producción de conocimiento- para concentrarse en canalizar los casos más graves y promover entre la población local un nuevo lenguaje que ayude a generar conciencia sobre la relación salud/enfermedad y su prevención. Entonces, ¿cómo hacer para aprovechar los conocimientos y saberes locales para el propósito científico? En este punto, justamente, conviene repensar los centros de atención en zonas rurales e indígenas para convertirlos en centros de investigación interdisciplinar e incrementar la participación tanto de profesionales como de personas interesadas en promover buenas prácticas de salud. De entrada, se contribuiría con ellos a que el médico considerara consultar a interlocutores profesionales en el análisis de casos en los que sea pertinente comprender un lenguaje distinto al de la ciencia médica. Las alianzas con organizaciones de médicos indígenas tradicionales, la creación de huertos medicinales de traspatio y el análisis de casos para entender los alcances y límites de las diferentes modalidades de atención aún están pendientes. También queda pendiente formalizar una serie de temas, como la epidemiología sociocultural, que permite incluir a la población en general en la toma de decisiones y el reconocimiento de problemas sanitarios, e invita a los profesionales de la salud a analizar el efecto de la intervención (Hersch-Martínez, 2013), ya que la constante es la realización de diagnósticos clínicos sin dar cabida al análisis etiológico de los diferentes padecimientos, desde la perspectiva de los distintos actores locales (Menéndez, 1998; Haro, 2010). 


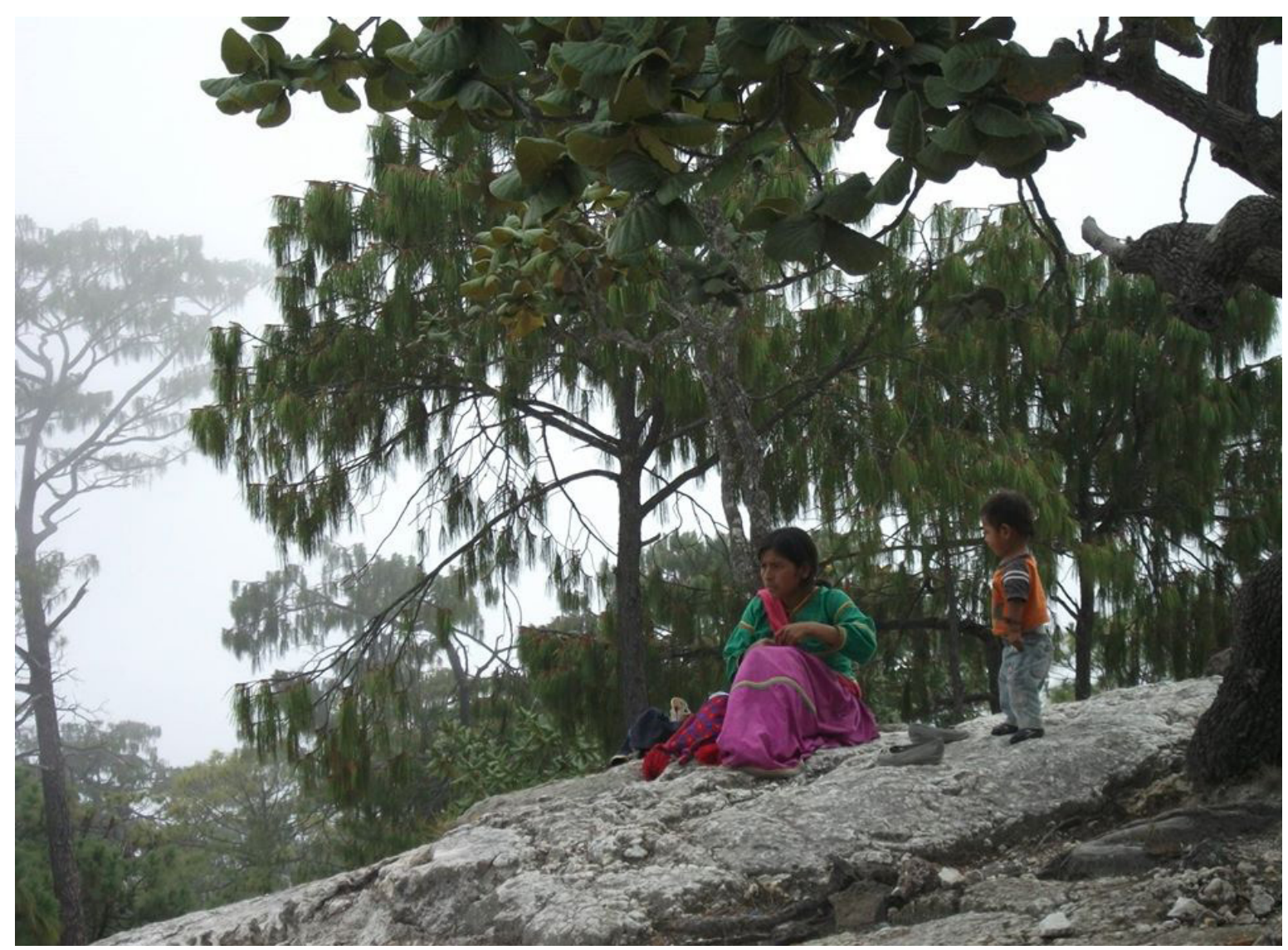

Gabriel CABezAS Mujer y niño descansando. Mezquitic, Jalisco, México.

\section{Conclusiones}

La interculturalidad en salud, en particular en las clínicas rurales — que operan bajo la misma lógica que cualquier otro consultorio médico de un hospital público, excepto por la carencia de equipos-, es un discurso que no encuentra prácticas definidas, y por lo tanto, se convierte en una falacia vigente que se sustenta en derechos universales. El interés por la salud se da desde diferentes modalidades de atención, que surgen de diferentes formas de vida y juegos de lenguaje (Jacorzynski, 2008), pero que están decididas a la interacción en tanto comparten el mismo espacio y tiempo. La divergencia puede diluirse y los centros de salud y clínicas rurales pueden convertirse en espacios de encuentro, más que de desencuentro, con la ciencia médica. La propuesta básica es no dar por sentado que el médico residente es el último responsable, sino entender que se encuentra en una etapa que requiere acompañamiento e interlocución con expertos de diferentes disciplinas vinculadas directa e indirectamente a la salud. Es necesario dejar de ver a las clínicas rurales como espacios de paso para subir en el escalafón y concebirlas como espacios de investigación locales, y de intercambio regional, para lo cual no se requiere un presupuesto especial sino participación coordinada, interinstitucional e interdisciplinaria. Para esto podrían tomarse dos o tres centros como proyectos piloto para el diseño 
de una nueva política pública mucho más incluyente. Hay que partir desde cero en este sentido, si se desea mejorar la calidad de la atención, favorecer la mutua comprensión y contribuir a que la ciencia médica erradique fenómenos como la brujería como explicación causal de padecimientos que requieren un segundo y hasta un tercer nivel de atención; y al mismo tiempo, permitir que las prácticas locales contribuyan a una mejora sustantiva de salud cuando esto sea posible, sin que medie el cuestionamiento propio de la demostración científica, como puede ser, por ejemplo, la contribución a la salud mental de las ofrendas de carácter ritual. En principio, expertos de diferentes disciplinas deberían analizar en conjunto casos particulares y generar líneas de investigación interdisciplinar de manera colegiada para convertir a la salud pública en un espacio efectivamente público. D

\section{Bibliografía}

Almaguer, José, Vicente Vargas y Hernán García (coords.), 2014, Interculturalidad en salud. Experiencias y aportes para el fortalecimiento de los servicios de salud, Secretaría de Salud/Gobierno de la República, México. Disponible en línea: <http://www.dgplades.salud.gob.mx/ Contenidos/Documentos/MedicinaTradicional/InterculturalidadSalud.pdf>.

Barajas, Gabriela, 2016, “Prospera, Programa de Inclusión Social: ¿̇una nueva estrategia de atención a la pobreza en México?”, en Revista Gestión y Estrategia, núm. 50, México, pp. 103-119.

Broda, Johanna y Jorge Báez (coords.), 2001, Cosmovisión, ritual e identidad de los pueblos indígenas de México, Fondo de Cultura Económica/Consejo Nacional para la Cultura y las Artes, México.

Consejo Nacional de Evaluación de la Política de Desarrollo Social (Coneval), 2019, Metodología para la medición multidimensional de la pobreza en México, tercera edición, Consejo Nacional de Evaluación de la Política de Desarrollo Social, México.

Comaroff, Jean y John Comaroff, 2013, Teoría desde el Sur. O cómo los países centrales evolucionan hacia África, Siglo XXI Editores, Buenos Aires.

Diario Oficial de la Federación, 2014, Decreto por el que se crea la Coordinación Nacional de Prospera Programa de Inclusión Social, 5 de septiembre, México. Disponible en línea: <http://dof.gob.mx/nota_detalle.php?codigo=5359088\&fecha=05/09/2014>.

Dietz, Gunther, 2003, Multiculturalismo, interculturalidad y educación: una aproximación antropológica, Centro de Investigaciones y Estudios Superiores en Antropología Social/Universidad de Granada, Granada y México.

Flores, Daniel, 2012, El sentido social y cultural de los vivos en su relación con los muertos. El panteón de Romerillo, Chamula, Chiapas, tesis de maestría, Centro de Investigaciones y Estudios Superiores en Antropología Social, San Cristóbal de las Casas.

—_, 2017, (Des)encuentros de la ciencia médica con las prácticas de curación locales en la sierra de Aquismón, SLP”, tesis de doctorado, Centro de Investigaciones y Estudios Superiores en Antropología Social, México.

Gallardo, Juan, 2005, Medicina tradicional P’urhépecha, El Colegio de Michoacán/Universidad Cultural Indígena de Michoacán, Zamora.

Gallardo, Patricia, 2004, “Los especialistas de la curación. Curanderos teenek y nahuas de Aquismón”, en Anales de Antropología, vol. 38, núm. 1, pp. 179-200.

García Canclini, Néstor, 2004, "Introducción: teorías de la interculturalidad y fracasos políticos", en Diferentes, desiguales y desconectados. Mapas de la interculturalidad, Gedisa, Barcelona, pp. 13-26.

(coord.), 2011, Conflictos interculturales, Gedisa, Barcelona.

González Muñoz, Verónica Alejandra y Martha Oliva Zárate, 2016, "El federalismo en México: principios generales y distribución de competencias frente a la política social de México: el caso del programa Prospera (2016)", en Revista Ciencia Administrativa, núm. 2, pp. 1-10.

Haro, Jesús Armando (org.), 2010, Epidemiología sociocultural. Un diálogo en torno a su sentido, métodos y alcances, El Colegio de Sonora/ Lugar Editorial, Hermosillo-Buenos Aires.

Hersch-Martínez, Paul, 2013, “Epidemiología sociocultural: una perspectiva necesaria”, en Salud Publica de México, núm. 55, pp. 512-518.

Instituto Mexicano del Seguro Social (IMSs), s.f., Informe del programa ImSs-Oportunidades. Disponible en línea: <http://www.imss.gob.mx/ sites/all/statics/pdf/informes/20132014/12_Cap08.pdf> 
Jacorzynski, Witold, 2008, En la cueva de la locura: aportación de Ludwig Wittgenstein a la antropología social, Centro de Investigaciones y Estudios Superiores en Antropología Social, México.

——, 2010 La maldición de Judas Iscariote: aportación de Ludwig Wittgenstein a la teología, la filosofía y la antropología de la religión, Centro de Investigaciones y Estudios Superiores en Antropología Social, México.

Lear, Jonathan, 2006, Radical Hope. Ethics in the Face of Cultural Devastation, Harvard University Press, Cambridge.

Lerín, Sergio, 2004, "Antropología y salud intercultural: desafíos de una propuesta”, en Desacatos. Revista de Ciencias Sociales, núms. 15-16, pp. 111-125.

López Austin, Alfredo, 1972, "El mal aire en el México prehispánico", en Jaime Litvak King y Noemí Castillo Tejero (eds.), Religión en Mesoamérica, XII Mesa Redonda, Sociedad Mexicana de Antropología/Universidad de las Américas en Cholula, México, pp. $399-408$.

— 1984 , Cuerpo humano e ideología: las concepciones de los antiguos nahuas, Universidad Nacional Autónoma de México (Serie Antropológica, 39), México.

Menéndez, Eduardo, 1998, "Estilos de vida, riesgos y construcción social. Conceptos similares y significados diferentes", en Estudios Sociológicos, vol. XVI, núm. 46, pp. 37-67.

—_, 2006, "Interculturalidad, 'diferencias' y antropología at home. Algunas cuestiones metodológicas”, en Gerardo Fernández (coord.), Salud e interculturalidad en América Latina. Antropología de la salud y crítica intercultural, Abya-Yala, Quito, pp. 51-65.

__ 2016, "Intercultural Health: Proposals, Actions and Failures", en Ciência e Saúde Coletiva, vol. 21, núm. 1, pp. 109-118.

Menéndez, Eduardo y Renée di Pardo, 2009, Miedos, riesgos e inseguridades. Los medios, los profesionales y los intelectuales en la construcción social de la salud como catástrofe, Centro de Investigaciones y Estudios Superiores en Antropología Social, México.

Organización Panamericana de Salud (OPS), 1993, Resolución CD37.R5. Salud de los pueblos indigenas, Washington, D. C.

- 2008, Una visión de salud intercultural para los pueblos indigenas de las Américas, Washington, D. C.

Tomasini, Alejandro, 2014, Tópicos wittgensteinianos, Edere, México.

Tomlinson, John, 2001, Globalización y cultura, Oxford University Press, México.

Wittgesntein, Ludwig, 1999, Investigaciones filosóficas, Ediciones Altaya, España.

Zolla, Carlos, 2011, "Del Imss-Coplamar a la experiencia del Hospital Mixto de Cuetzalan. Diálogos, asimetrías e interculturalidad médica”, en Arturo Argueta, Eduardo Corona y Paul Hersch (coords.), Saberes colectivos y diálogo de saberes en México, Centro Regional de Investigaciones Multidisciplinarias-Universidad Nacional Autónoma de México/Universidad Iberoamericana, Puebla, pp. $201-231$.

\section{Entrevistas}

Felipe González, médico residente, Tanzozob, Aquismón, San Luis Potosí, 28 de julio, 7 de agosto y 12 de agosto de 2014. 